\title{
Hunting for the Adhesion Molecule, Retinoschisin, in Retina using CEMOVIS
}

J. Bernard Heymann ${ }^{1 *}$, Christopher K. E. Bleck ${ }^{2}$, Robert N. Fariss ${ }^{3}$, Camasamudram Vijayasarathy ${ }^{4}$, Dennis C. Winkler ${ }^{1}$, Rick Huang ${ }^{5}$, Altaira D. Dearborn ${ }^{1}$, Aleksandr Smirnov ${ }^{3}$, Paul A. Sieving ${ }^{4,6}$ and Alasdair C. Steven ${ }^{1}$

1. Laboratory for Structural Biology Research, NIAMS, NIH, Bethesda, MD, USA.

2. Electron Microscopy Core, NHLBI, NIH, Bethesda, MD, USA.

${ }^{3 .}$ Biological Imaging Core, NEI, NIH, Bethesda, MD, USA.

4. Section on Translational Research for Retinal and Macular Degeneration, NIDCD, NIH, Bethesda, MD, USA.

5. Cryo-Electron Microscopy Facility, Janelia Research Campus, HHMI, Ashburn, VA, USA.

6. NEI, NIH, Bethesda, MD, USA.

* Corresponding author: heymannb@mail.nih.gov

In studying biologically relevant molecules, the ultimate aim is to visualize them in their native environment. One technique that has the potential to resolve individual molecules in cells and tissues is cryo-electron microscopy of vitreous sections (CEMOVIS [1] [2]) combined with cryo-electron tomography (cryo-ET). We are interested in revealing the disposition of the retinal adhesion protein, retinoschisin (RS1), within the retina. RS1 is secreted into the intercellular spaces between the inner segments of photoreceptors, and between bipolar cells, shown by immuno-fluorescence (Figure 1) and immuno-electron microscopy [3]. We regard RS1 as an adhesion molecule that contributes to the integrity of the layered retinal architecture. Loss-of-function mutations in RS1 cause X-linked retinoschisis (XLRS), a debilitating disease associated with the formation of retinal cavities or cysts (schisis), eventually leading to vision loss in young males. We solved the structure of RS1 at $4.1 \AA$ resolution by cryo-electron microscopy (cryo-EM), visualizing a 16-mer composed of two back-to-back octameric rings with a combined mass of $\sim 380 \mathrm{kDa}$ (Figure $2 \mathrm{~A}, \mathrm{~B}$ ) [4]. From this structure it is clear that many mutations in the subunit interfaces would preclude assembly of the rings. However, other mutations at the periphery of the rings also cause disease, but it was unclear how. In further cryo-EM experiments, we have observed that RS1 double rings self-assemble into extensive branched networks (Figure 2C) [5]. We identified an important peripheral loop involved in interaction between these molecules. Mutations in this loop decrease or prevent secretion, suggesting that higher order assembly may occur before secretion and is relevant to the disease. To ascertain whether similar assemblies also occur in situ, we started to examine mouse retinal tissue using CEMOVIS and cryo-electron tomography. We are facing several technical challenges in tissue preparation, sectioning and data acquisition, although none of them are considered insurmountable. As an illustration of what we can achieve with CEMOVIS, we have obtained images of 50-70 nm cryosections that show clear distinction of microtubular protofilaments separated by $\sim 5 \mathrm{~nm}$ (Figure $3 \mathrm{~A}$ ). We performed cryo-ET of purified RS1, showing that the double-ring structure of RS1 is readily observable in tomographic sections (Figure 3B). We therefore believe this approach can tell us whether RS1 assembles into networks in the intercellular space between photoreceptors and bipolar cells.

\section{References:}

[1] AW McDowall et al., J Microsc 131 (1983), p. 1.

[2] A Al-Amoudi et al., Nature 450 (2007), p. 832.

[3] C Vijayasarathy et al., Invest Ophthalmol Vis Sci 48 (2007), p. 991. 
[4] G Tolun et al., Proc Natl Acad Sci U S A 113 (2016), p. 5287.

[5] JB Heymann et al., J Cell Biol (2019), ePub: 2019/01/12.

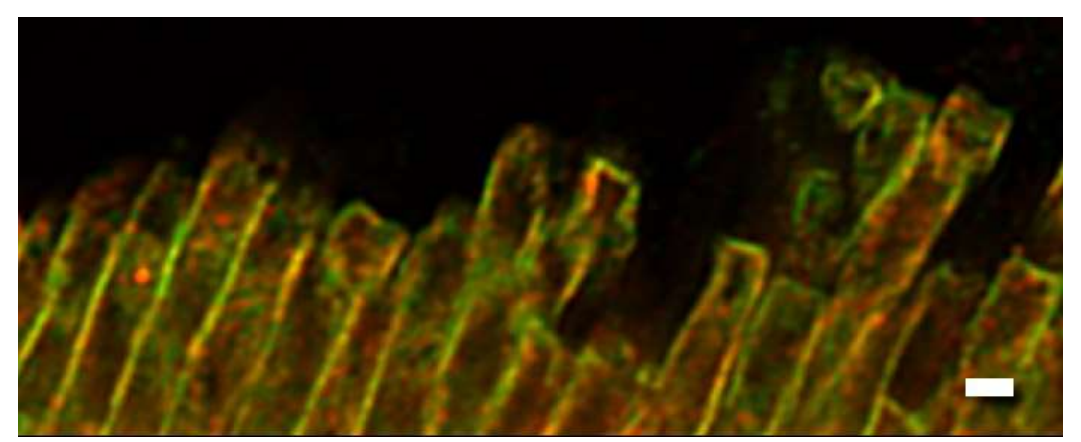

Figure 1. Vibratome section of aldehyde-fixed mouse retina immunolabeled with antibodies to RS1 + goat anti-rabbit Alexa 488 (green) and NaK-ATPase + goat anti-mouse Alexa 555 (red) imaged on a Zeiss 880 Airyscan microscope with a 63x 1.4NA objective. Both RS1 and NaK-ATPase are abundant along the cylindrical profiles of the photoreceptor inner segments, but co-localization is difficult to judge. Scale bar: $1 \mu \mathrm{m}$.
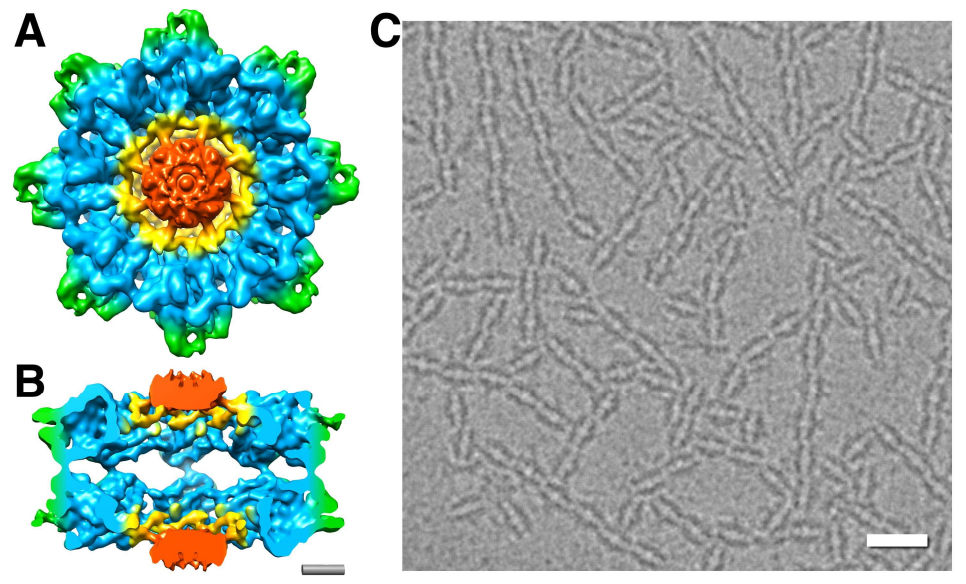

Figure 2. (A,B) Cryo-EM structure of retinoschisin at $4.1 \AA$ (EMD-6425) (Polara at $300 \mathrm{kV}$ with a K2 detector). Scale bar: $2 \mathrm{~nm}$. (C) Cryo-EM of a network of RS1 filaments (Krios at $300 \mathrm{kV}$ with a K2 detector). Scale bar: $20 \mathrm{~nm}$.

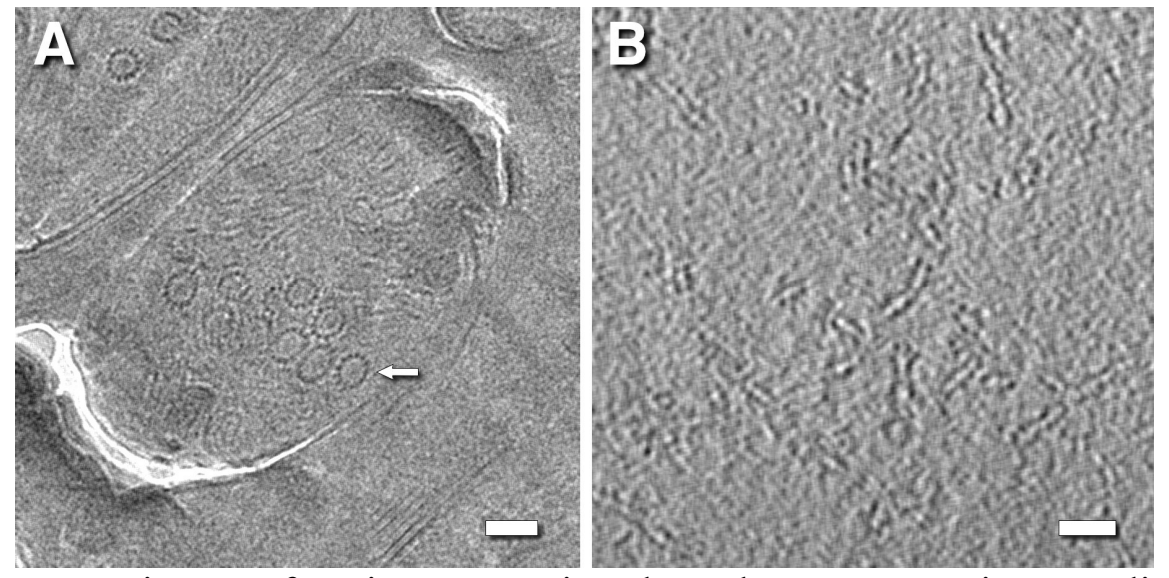

Figure 3. (A) Cryo-EM image of a vitreous section through a mouse retina ganglion axon, showing microtubules with protofibrils at $\sim 5 \mathrm{~nm}$ separation (JEOL 2200 at $200 \mathrm{kV}$ with a K2 detector). Scale bar: $50 \mathrm{~nm}$. (B) Slice through a $27 \mathrm{~nm}$ thick tomogram of purified RS1, showing separation of the double rings at $\sim 5 \mathrm{~nm}$ (Krios at $300 \mathrm{kV}$ with a K2 detector). Scale bar: $20 \mathrm{~nm}$. 